\section{Isolation of Listeria monocytogenes in a salami producing plant in Piedmont: use of pulsed field gel electrophoresis to trace contaminations}

\author{
Annalisa Costa, Maria Ausilia Grassi, \\ Sara Lomonaco, Patrizia Morra, \\ Daniele Nucera, Tiziana Civera \\ Dipartimento di Patologia Animale, \\ Facoltà di Medicina Veterinaria, \\ Università degli Studi di Torino, \\ Grugliasco, Italy
}

\section{Abstract}

The ability of Listeria monocytogenes to survive in different environments and establish persistent contaminations is an important issue for food producers. This study aimed to assess the environmental contamination level in an Italian salami producing plant and to identify possible sources of contamination using pulsed field gel electrophoresis (PFGE) on $L$. monocytogenes isolates obtained from environmental $(n=54)$ and meat samples $(\mathrm{n}=9)$ collected over 9 months. Detection of $L$. monocytogenes was performed using the UN EN ISO 11290-1 procedure and every isolate was characterised with PFGE, using $A s c \mathrm{I}$ and $A p a I$ restriction enzymes. The environmental detection frequencies were constant both in the first (22\%) and the second (27\%) visit, thus suggesting the presence of strains adapted to the processing plant. Equipments can represent a reservoir of $L$. monocytogenes from which it can spread into the whole producing plant. The reservoir was documented by PFGE results which showed several persistent strains. Moreover, PFGE proved the cross-contamination between surfaces and semiprocessed products like pastes, which furthermore have been contaminated by L. monocytogenes in $100 \%$ of samples in the first two visits and in $33 \%$ in the last visit. This study gave evidence that detection methods and PFGE characterisation can be effective tools to detect possible sources and routes of contamination.

\section{Introduzione}

Listeria monocytogenes fa parte del genere Listeria ed è il principale patogeno tra le specie che ne fanno parte (Low e Donachie, 1997). Si tratta di un microrganismo a trasmissione prevalentemente alimentare ed è l'agente eziolo- gico della listeriosi, una patologia con un elevato impatto sulla salute pubblica a causa dell'elevato tasso di mortalità stimato intorno al $20-30 \%$. Le categorie di persone maggiormente suscettibili all'infezione sono i cosiddetti YOPI (young, old, pregnant, immuno-compromised), ovvero neonati, soggetti anziani, donne in gravidanza ed immunocompromessi (WHO/FAO, 2004).

Si tratta di un microrganismo capace di adattarsi a vari tipi di stress: è, infatti, un microrganismo psicrotrofo e alotollerante in grado di crescere in ampi intervalli di $\mathrm{pH}$ e fino a valori di water activity $\left(\mathrm{A}_{\mathrm{w}}\right)$ di 0,92 , con un valore ottimale di sviluppo a 0,99 (Low e Donachie, 1997). Tali proprietà rendono gli alimenti pronti al consumo [ready-to-eat (RTE)] una categoria di prodotti a rischio di trasmissione del patogeno poiché vengono conservati, anche per tempi abbastanza prolungati, a temperatura di refrigerazione e non subiscono ulteriori trattamenti termici in grado di abbattere la carica microbica prima del consumo. Una categoria di alimenti RTE che comprende una grande varietà di prodotti è rappresentata dai salumi. Durante la produzione degli insaccati fermentati si verificano delle modificazioni dei parametri intrinseci utili ad impedire lo sviluppo di microrganismi patogeni rendendoli sicuri. I salami arrivati a fine stagionatura presentano, infatti, pH di circa 5,3-6,2, $\mathrm{A}_{\mathrm{w}}$ di circa $0,82-0,85$ ed una concentrazione di sale intorno al 5\%. Tuttavia patogeni come Escherichia coli 0157:H7, Salmonella, Staphylococcus aureus e L. monocytogenes, grazie ad una contaminazione che può avvenire in tutte le fasi della catena produttiva (Reij e De Aantrekker, 2004), possono essere presenti e sopravvivere alle condizioni che si vengono ad instaurare durante il periodo di stagionatura (Leroy et al., 2006). Le possibili fonti di cross-contaminazione sono rappresentate dagli ingredienti utilizzati per la produzione dell'alimento, gli operatori che manipolano materie prime ed attrezzi o le superfici con cui vengono a contatto i prodotti (Samelis et al., 1998; Chasseignaux et al., 2001).

La presenza di $L$. monocytogenes nell'industria alimentare rappresenta, quindi, una criticità poiché questo microorganismo è in grado non solo di sopravvivere ma anche di trovare condizioni favorevoli alla crescita in ambienti freddi e umidi come pavimenti, canali di scolo ed attrezzature dei locali di produzione. Ceppi di L. monocytogenes sono stati isolati, infatti, da superfici a contatto con gli alimenti, anche qualora queste superfici fossero già state pulite e disinfettate regolarmente (Pan et al., 2006). La persistenza nell'ambiente potrebbe essere dovuta alla capacità di determinati ceppi di adattarsi rapidamente in funzione del tipo di stress a cui sono sottoposti o di aggregarsi in comunità cellulari dette biofilm, in cui i microrganismi aderiscono alle superfici e
Correspondence: Annalisa Costa, Dipartimento di Patologia Animale, Facoltà di Medicina Veterinaria, Università degli Studi di Torino, via Leonardo da Vinci 44, 10095 Grugliasco, Italy. Tel: +39.011.6709219 - Fax: +39.011.6709224. E-mail: annalisa.costa@unito.it

Key words: Listeria monocytogenes, PFGE, Persistence, Environment, Food safety.

Conflict of interests: the authors declare no potential conflict of interests.

Received for publication: 15 January 2013.

Revision received: 26 March 2013.

Accepted for publication: 2 April 2013.

This work is licensed under a Creative Commons Attribution 3.0 License (by-nc 3.0).

(C) Copyright A. Costa et al., 2013

Licensee PAGEPress, Italy

Italian Journal of Food Safety 2013; 2:e20

doi:10.4081/ijfs.2013.e20

sono inclusi in una matrice costituita prevalentemente da polisaccaridi (Gandhi e Chikindas, 2007). I microrganismi organizzati in biofilm possono disporre di maggiori concentrazioni di nutrienti, godono di una migliore protezione nei confronti degli stress ambientali (Donlan, 2001), e mostrano una maggiore resistenza ai sanificanti, disinfettanti ed agenti antimicrobici rispetto alle cellule in forma planctonica (Robbins et al., 2005). Tali microrganismi possono, quindi, colonizzare zone difficilmente raggiungibili dai sanificanti presenti nell'ambiente, quali ad esempio crepe, irregolarità, angoli e fondi ciechi dei macchinari, che costituiscono delle nicchie ben protette in cui il microrganismo può quindi sopravvivere, fungendo da reservoir per la diffusione di $L$. monocytogenes all'interno dell'azienda e rappresentando quindi un problema per la sicurezza alimentare.

Molti studi hanno dimostrato come la persistenza di tali ceppi si possa protrarre per mesi ed anni in differenti tipi di industrie come in stabilimenti di macellazione e laboratori di sezionamento (Giovannacci et al., 1999), nell'industria di trasformazione della carne (Chasseignaux et al., 2001; Peccio et al., 2003; Thévenot et al., 2005) in stabilimenti di lavorazione dei prodotti della pesca (Destro et al., 1996; Autio et al., 1999; Chen et al., 2010), ed in caseifici (Miettinen et al., 1999; Kabuki et al., 2004; Lomonaco et al., 2009). Ad oggi esistono differenti metodiche di caratterizzazione molecolare che possono essere utilizzate per evidenziare la presenza di ceppi persistenti e sporadici nell'ambiente e determinarne la correlazione. In particolare, la metodica d'elezione è la pulsed field gel electrophoresis (PFGE), considerata il metodo gold standard anche per 
lo svolgimento delle indagini epidemiologiche sui patogeni trasmessi dagli alimenti, tra cui $L$. monocytogenes.

Questo studio ha inteso valutare il livello di contaminazione ambientale da parte di $L$. monocytogenes in un salumificio di medie dimensioni con lo scopo di evidenziare, attraverso la caratterizzazione tramite PFGE, l'eventuale presenza di ceppi persistenti e la possibile fonte di tale contaminazione.

\section{Materiali e Metodi}

In questo studio sono stati analizzati 77 ceppi di L. monocytogenes, provenienti da un salumificio piemontese di medie dimensioni che produce diversi tipi di prodotti (salami, prosciutto cotto, salame cotto, cotechino, zampone, salsiccia fresca, lardo, pancetta). Sono state effettuate 3 visite nell'arco di 9 mesi (aprile, giugno e dicembre); durante ogni sopralluogo sono stati eseguiti 18 prelievi ambientali $(\mathrm{n}=54)$, tramite il metodo spongebag, su altrettante superfici a contatto con gli alimenti (per un totale di $500 \mathrm{~cm}^{2}$ per ogni superficie), attrezzature e macchinari (Figura 1). I prelievi ambientali sono stati eseguiti in seguito alla sanificazione prevista al termine della produzione. Inoltre, durante ogni visita sono stati prelevati 3 campioni di impasto $(n=9)$ stoccati nella cella di riposo e destinati alla produzione di salami rosa, capriccio, crespone, filzetta e bocconcini, le tipologie di salumi più rappresentative della produzione del salumificio. I campioni sono stati prelevati in punti diversi del vagonetto in cui era stoccato l'impasto in modo da ottenere un campionamento uniforme.

L'isolamento di $L$. monocytogenes è stato realizzato utilizzando la procedura UNI EN ISO 11290-1 (ISO, 2005) che prevede il passaggio dei campioni in brodo di arricchimento primario (Demi Fraser-0xoid) e secondario (Fraser0xoid) e la successiva semina sui terreni solidi selettivi Palcam e OCLA (Oxoid). Dopo $48 \mathrm{~h}$ di incubazione a $37^{\circ} \mathrm{C}$, sono state isolate, ove possibile, 5 colonie con morfologia tipica $(\mathrm{n}=77)$ ed identificate tramite polymerase chain reaction (PCR) specie-specifica per $L$. monocytogenes, con amplificazione del frammento del gene prfA (D’Agostino et al., 2004).

Per la tipizzazione tramite PFGE, il DNA genomico di $L$. monocytogenes è stato incluso in blocchetti di agarosio come descritto da Graves e Swaminathan (2001) e successivamente digerito con due enzimi di restrizione: AscI e ApaI (New England Biolabs, Beverly, MA, USA). I profili genomici generati sono stati comparati mediante il software Bionumerics (v 4,0; Applied Maths, Kortrijk, Belgio) e la similitudine tra i profili è stata determinata attraverso il coefficiente di Dice, utilizzando valori di ottimizzazione pari all' $1,2 \%$ per $A s c \mathrm{I}$ e all'1,4\% per ApaI e valori di tolleranza di posizione pari all'1,4\% per entrambi gli enzimi.

I risultati di ogni enzima sono stati raccolti in una matrice, combinati tra loro ed utilizzati per generare un dendrogramma utilizzando l'algoritmo unweighted pair group method with arithmetic mean (UPGMA). I pulsotipi sono stati considerati identici quando i loro pattern erano indistinguibili, e correlati quando il coefficiente di Dice era maggiore del $90 \%$.

\section{Risultati}

Nel corso delle tre visite al salumificio sono stati raccolti complessivamente 54 campioni ambientali e 9 campioni di diversi tipi di impasto. Nove campioni ambientali su 54 sono risultati positivi per $L$. monocytogenes $(16,6 \%)$ e 12 per Listeria spp. (22,2\%) (Tabella 1). Il $38,9 \%$ delle superfici campionate è risultato contaminato da L. monocytogenes e l'andamento delle positività ambientali è rimasto costante nei mesi di aprile e giugno, rispettivamente con 4 positività su 18 campioni $(22,2 \%)$ e 5 su 18 (27,7\%). Al contrario, nella visita di dicembre non sono state riscontrate positività. Per quanto riguarda Listeria spp. si sono osservate 6 positività su 18 campioni (33,3\%) durante la prima visita, il valore è sceso a 4 su $18(22,2 \%)$ a giugno e a 2 su 18 a dicembre (11\%).

Per quanto riguarda gli impasti prelevati, 7 di questi sono risultati contaminati con $L$. monocytogenes, mentre tutti contenevano Listeria spp. In particolare, tutti gli impasti prelevati ad aprile e giugno contenevano L. monocytogenes e Listeria spp., mentre a dicembre la contaminazione si è abbassata ad una positività su 3 prelievi per L. monocytogenes; i campioni risultati negativi sono rimasti, però, positivi per Listeria spp.

Tutte le colonie isolate mediante la ricerca qualitativa su piastra $(n=77)$ sono risultate appartenere alla specie $L$. monocytogenes e tipizzabili mediante PFGE, con entrambi gli enzimi utilizzati. La combinazione dei pattern generati dai due enzimi ha permesso di identificare 64 pulsotipi, con una percentuale mini-

Tabella 1. Positività per L. monocytogenes e Listeria spp. registrate nel corso dei tre sopralluoghi effettuati e numero di colonie di $L$. monocytogenes isolate da ogni sito/tipo di impasto contaminato dal microrganismo.

\begin{tabular}{|c|c|c|c|c|c|c|c|}
\hline \multirow[t]{2}{*}{ Prelievi ambientali } & \multicolumn{2}{|c|}{ Aprile } & \multicolumn{2}{|c|}{ Giugno } & \multicolumn{2}{|c|}{ Dicembre } & \multirow{2}{*}{$\begin{array}{c}\text { Colonie } \\
\text { di } L . m \text {. isolate }\end{array}$} \\
\hline & L. spp. & L. m. & L. spp. & L. m. & L. spp. & L. m. & \\
\hline \multicolumn{8}{|l|}{ Macchinari } \\
\hline Testa tritacarne & + & - & + & + & + & - & 5 \\
\hline Nastro n. 2 & - & + & - & - & - & - & 2 \\
\hline Vagonetto & + & + & - & - & - & - & 5 \\
\hline Scotennatrice & - & - & - & - & + & - & - \\
\hline Insaccatrice 1 - area di uscita & + & + & + & + & - & - & 9 \\
\hline Insaccatrice 1 - parti interne & - & - & + & + & - & - & 1 \\
\hline Insaccatrice 2 - area di uscita & + & - & - & - & - & - & - \\
\hline Pungibudello & + & + & + & + & - & - & 8 \\
\hline Legatrice - arricciabudelli & + & - & + & + & - & - & 7 \\
\hline \multicolumn{8}{|l|}{ Impasti } \\
\hline Salame rosa & + & + & + & + & + & + & 15 \\
\hline Salame capriccio & + & + & + & + & + & - & 8 \\
\hline Salame crespone & + & + & np & np & 8 & & \\
\hline Salame filzetta & np & + & + & np & 9 & & \\
\hline Bocconcini & np & np & + & - & - & & \\
\hline
\end{tabular}

L. m., L. monocytogenes; L. spp., Listeria species; $\mathrm{np}$, non prelevato. 
ma di similitudine del $46,2 \%$. I ceppi sono stati suddivisi in 54 pulsotipi unici e 10 condivisi, sette dei quali che raggruppano pattern generati da ceppi provenienti da diversi tipi di impasto. Inoltre, è stata evidenziata la presenza di 12 cluster con percentuale di similitudine superiore o uguale al 90\% (Figura 2), la cui composizione è mostrata in Tabella 2.

\section{Discussione}

La normativa europea, ed in particolare il Regolamento CE 178/2002 (Commissione Europea, 2002), indica l'operatore del settore alimentare (OSA) come il principale responsabile della sicurezza degli alimenti prodotti e trasformati all'interno della propria azienda. Egli è tenuto ad elaborare, applicare ed aggiornare un piano di autocontrollo in modo da garantire la salubrità degli alimenti in ogni fase della lavorazione. In questo contesto, per uno stabilimento di trasformazione delle carni, è molto importante mettere in atto un programma di controllo ambientale volto a monitorare la presenza e la diffusione di patogeni come $L$. monocytogenes nei locali di produzione. La ricerca di $L$. monocytogenes è utile per definire possibili hot spot (siti a maggiore frequenza di isolamento) che potrebbero essere responsabili di eventuali contaminazioni del prodotto, mentre l'impiego di metodi di tipizzazione molecolare, come la PFGE, può identificare i ceppi responsabili di tale colonizzazione. Questo consente di individuare eventuali criticità legate a ceppi disseminati all'interno della realtà esaminata in grado di persistere nell'ambiente per mesi o addirittura per anni.

La ricerca di $L$. monocytogenes negli ambienti di lavorazione del salumificio oggetto di studio ha permesso di evidenziare in tutti i sopralluoghi effettuati una maggior frequenza di isolamento di Listeria spp. rispetto a $L$. monocytogenes. Questo potrebbe indicare una maggior diffusione di Listeria spp. nell'ambiente rispetto a $L$. monocytogenes. Occorre sottolineare come l'isolamento di Listeria spp. possa essere considerato un indicatore della presenza di $L$. monocytogenes; dati sperimentali hanno infatti dimostrato come il $40 \%$ dei campioni positivi per Listeria spp. fosse anche positivo per L. monocytogenes (Tompkin, 2002). Elevate prevalenze di Listeria spp. possono, quindi, indicare la possibilità che i locali di produzione possano essere anche contaminati da $L$. monocytogenes, in considerazione di caratteristiche di crescita e sopravvivenza molto simili.

La ricerca di $L$. monocytogenes negli ambienti di lavorazione del salumificio ha evidenziato la presenza di ceppi adattatisi all'ambiente nonostante le quotidiane procedure di sanificazione, che prevedevano l'applicazione di un sanificante schiumogeno alcalino clorato al termine della lavorazione ed il suo risciacquo prima dell'inizio della produzione il giorno successivo. L'andamento delle positività in seguito alle prime due visite è, infatti, rimasto costante. La contaminazione di superfici a contatto con gli alimenti (quali ad esempio tritacarne, nastro trasportatore, vagonetto, scotennatrice, legatrice e pungibudello) rappresenta una criticità poiché interi lotti di prodotto potrebbero andare incontro a cross-contaminazione. Basti pensare all'epidemia che nel 2008 in Canada causò 57 casi di listeriosi e 22 vittime associata ad un reservoir di $L$. monocytogenes, localizzato a livello di un'affettatrice in un noto stabilimento produttore di specialità RTE (Weatherill, 2009). La presenza di $L$. monocytogenes in queste tipologie di siti di prelievo potrebbe essere dovuta alla loro struttura complessa, ricca di angoli e fondi ciechi, difficilmente raggiungibili durante le operazioni di sanificazione. Anche nel corso dei campionamenti svolti nella nostra indagine, infatti, è stato problematico strofinare le spongebag in modo uniforme a causa della struttura dei macchinari. Inoltre, a livello di arricciabudello e pungibudello erano presenti residui di materiale organico, indice che la detersione non è stata efficace. Il tritacarne e l'insaccatrice presentavano ristagni di acqua, potenzial-

Tabella 2. Composizione e data di isolamento dei cluster con percentuale di similitudine superiore o uguale al $90 \%$, ottenuti dalla combinazione dei risultati pulsed field gel electrophoresis generati dagli enzimi di restrizione $A s c \mathrm{I}-A p a \mathrm{I}$.

\begin{tabular}{|c|c|c|c|c|}
\hline & & $A s c$ & & \\
\hline Cluster & Campioni (n) & Similitudine (\%) & $\begin{array}{l}\text { Campione di } \\
\text { provenienza }\end{array}$ & Data \\
\hline 1 & 6 & 91,2 & $\begin{array}{l}\text { I-Salame rosa } \\
\text { I-Salame rosa } \\
\text { I-Salame capriccio } \\
\text { I-Salame capriccio } \\
\text { S-Pungibudello } \\
\text { S-Pungibudello } \\
\text { I-Salame crespone }\end{array}$ & $\begin{array}{l}27 / 4 \\
22 / 6 \\
27 / 4 \\
22 / 6 \\
27 / 4 \\
22 / 6 \\
27 / 4\end{array}$ \\
\hline 2 & 2 & 95,8 & $\begin{array}{l}\text { S-Pungibudello } \\
\text { S-Testa tritacarne }\end{array}$ & $\begin{array}{l}27 / 4 \\
22 / 6\end{array}$ \\
\hline 3 & 7 & 92,4 & $\begin{array}{l}\text { I-Salame rosa } \\
\text { I-Salame capriccio } \\
\text { I-Salame crespone } \\
\text { S-Pungibudello }\end{array}$ & $\begin{array}{l}2 / 12 \\
22 / 6 \\
27 / 4 \\
22 / 6\end{array}$ \\
\hline 4 & 6 & 95,8 & $\begin{array}{l}\text { I-Salame rosa } \\
\text { I-Salame capriccio } \\
\text { I-Salame crespone } \\
\text { S-Pungibudello }\end{array}$ & $27 / 4$ \\
\hline 5 & 3 & 98,2 & $\begin{array}{l}\text { I-Salame filzetta } \\
\text { I-Salame rosa } \\
\text { I-Salame capriccio }\end{array}$ & $22 / 6$ \\
\hline 6 & 3 & 91 & $\begin{array}{l}\text { I-Salame crespone } \\
\text { I-Salame rosa } \\
\text { I-Salame capriccio }\end{array}$ & $27 / 4$ \\
\hline 7 & 2 & 95 & $\begin{array}{l}\text { I-Salame crespone } \\
\text { I-Salame rosa }\end{array}$ & $27 / 4$ \\
\hline 8 & 3 & 97,5 & $\begin{array}{l}\text { I-Salame capriccio } \\
\text { S-Pungibudello } \\
\text { S-Testa tritacarne }\end{array}$ & $\begin{array}{l}27 / 4 \\
27 / 4 \\
22 / 6\end{array}$ \\
\hline 9 & 2 & 90 & $\begin{array}{l}\text { I-Salame crespone } \\
\text { S-Vagonetto }\end{array}$ & $27 / 4$ \\
\hline 10 & 2 & 92,9 & $\begin{array}{l}\text { I-Salame crespone } \\
\text { S-Vagonetto }\end{array}$ & $27 / 4$ \\
\hline 11 & 4 & 95,7 & $\begin{array}{l}\text { I-Salame rosa } \\
\text { I-Salame rosa } \\
\text { I-Salame capriccio } \\
\text { S-Pungibudello }\end{array}$ & $\begin{array}{l}27 / 4 \\
22 / 6 \\
27 / 4 \\
27 / 4\end{array}$ \\
\hline 12 & 2 & 94,4 & $\begin{array}{l}\text { S-Insaccatrice } 1 \text { (area uscita) } \\
\text { S-Insaccatrice } 1 \text { (parti interne) }\end{array}$ & $22 / 6$ \\
\hline
\end{tabular}

I, impasto; S, spongebag. 


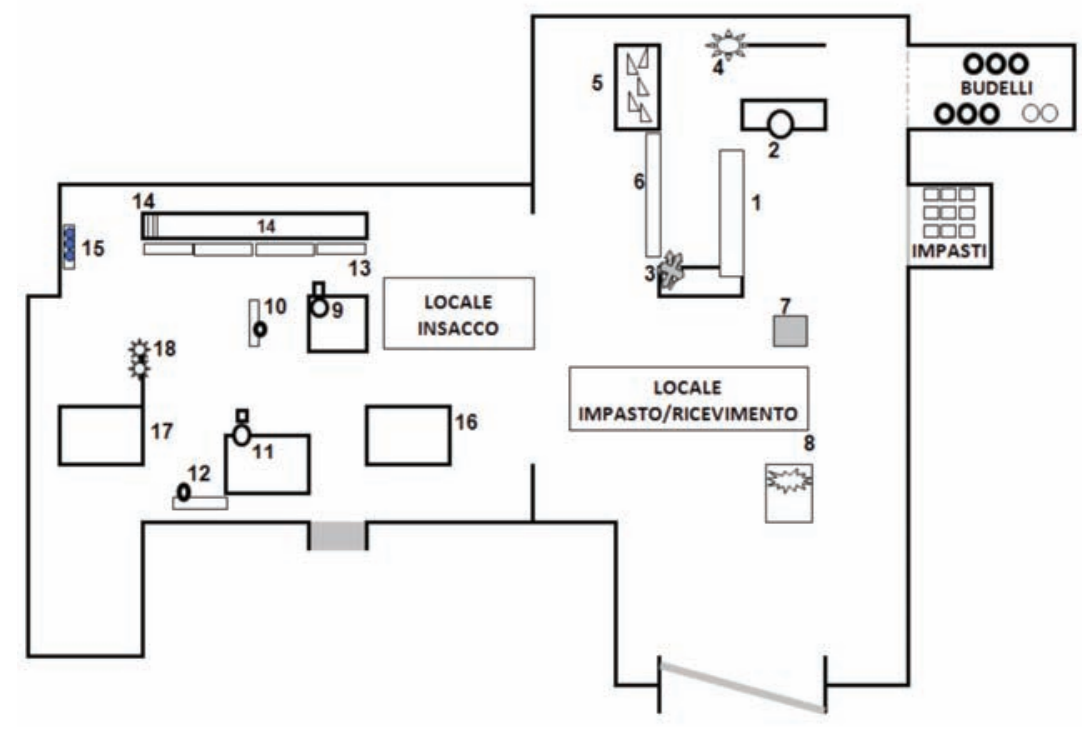

Figura 1. Piantina dello stabilimento e punti di prelievo dei campioni ambientali: 1, nastro - linea tritacarne-impastatrice; 2 , testa tritacarne; 3 , impastatrice - giunzione albero; 4, cutter; 5 , impastatrice - palette; 6 , nastro 2 - linea tritacarne-impastatrice; 7 , vagonetto; 8 , scotennatrice; 9 , insaccatrice 1 - area di uscita; 10 , insaccatrice 1 - parti interne; 11 , insaccatrice 2 - area di uscita; 12 , insaccatrice 2 - parti interne; 13 , tavolo insacco salami grossi; 14, nastro insacco salami grossi; 15 , pungibudello; 16, tavolo carne fresca; 17 , tavolo insacco salami piccoli; 18, legatrice - arricciabudelli.

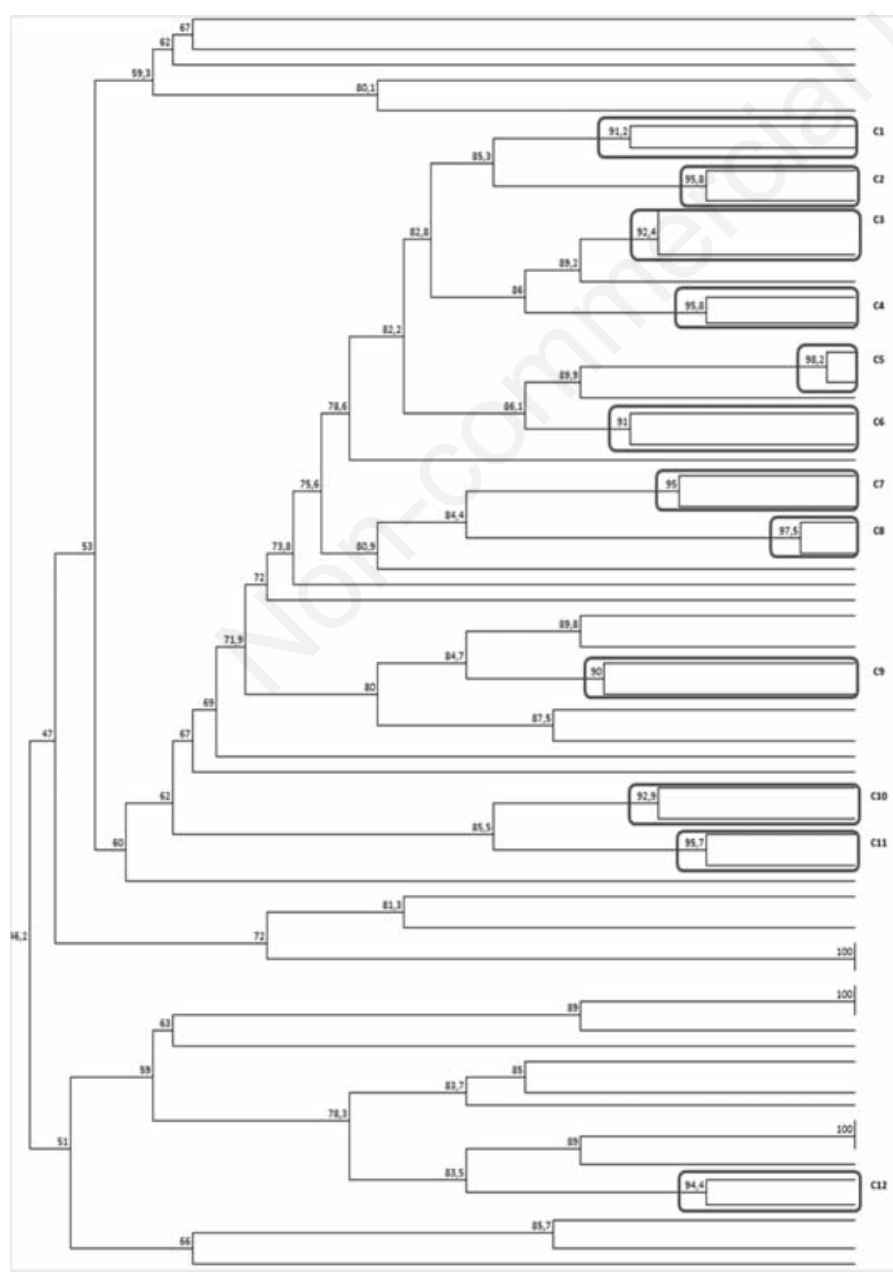

Figura 2. Rappresentazione schematica del dendrogramma generato dalla combinazione dei risultati dei due enzimi. I box evidenziano $i$ cluster indicati in Tabella 2. mente correlati ad una ridotta azione dei disinfettanti utilizzati. Questi rilievi sono di particolare criticità se si considera l'abilità di $L$. monocytogenes di formare biofilm e di sviluppare resistenza ai sanificanti (Palmer et al., 2007).

La struttura dei locali di lavorazione gioca un ruolo importante nella disseminazione del microrganismo: in un flusso di produzione che non segue il principio della marcia in avanti, la presenza di spazi aperti aumenta il rischio di cross-contaminazione (Reij e De Aantrekker, 2004). Il salumificio oggetto di studio è composto da un unico ampio locale in cui avviene la produzione di differenti tipi di specialità, che crea condizioni particolarmente favorevoli per le cross-contaminazioni. In considerazione dei risultati sfavorevoli delle prime due visite, il produttore ha deciso di modificare le modalità di sanificazione, sostituendo il prodotto clorato con un sanificante a base di policloruro di biguanide, e di migliorare la formazione del personale che le esegue. Come conseguenza, le frequenze di Listeria spp. sono diminuite sia per quanto riguarda gli impasti che le superfici di lavorazione, mentre $L$. monocytogenes non è stata più ritrovata a livello ambientale e le contaminazioni degli impasti sono diminuite (si è passati dalla contaminazione della totalità degli impasti ad una positività su tre campioni). Tuttavia, il nuovo sanificante introdotto ha causato il deterioramento di alcune superfici, cambiandone il colore e favorendone l'arrugginimento, ed il produttore ha deciso di ripristinare l'utilizzo del prodotto clorato e di alternarlo periodicamente con il biguanide. Questi risultati sono sovrapponibili a quanto riportato in letteratura: differenti autori hanno dimostrato, infatti, che l'eliminazione di $L$. monocytogenes è possibile migliorando le modalità di sanificazione (Autio et al., 1999; Miettinen et al., 1999). Considerando quindi i risultati ottenuti, si potrebbe ipotizzare la presenza di un reservoir di $L$. monocytogenes nella prima parte del salumificio (locale ricevimento/impasto). I campioni derivanti dal tritacarne e dalla scotennatrice, localizzati in quell'area erano infatti positivi per Listeria spp. e potrebbero quindi aver svolto la funzione di vettore di L. monocytogenes. Tuttavia quest'ultima potrebbe essere stata introdotta con la carne in ingresso nello stabilimento, come documentato da differenti studi (Chasseignaux et al., 2001; Peccio et al., 2003; Thévenot et al., 2005). Inoltre, la pancetta utilizzata nel salumificio è provvista di cotenna, che può veicolare $L$. monocytogenes in caso di contaminazione superficiale del suino. Questo potrebbe spiegare come, anche in seguito all'adozione di un piano di sanificazione più mirato, la scotennatrice sia risultata positiva alla presenza di Listeria spp., probabilmente reintrodotta con l'arrivo di una materia prima contaminata. Di conseguenza, per confermare questa ipotesi 
sarebbe necessario svolgere ulteriori studi volti ad analizzare le materie prime introdotte nel salumificio e svolgere sopralluoghi nei locali di lavorazione dei macelli fornitori.

L'applicazione della PFGE ha permesso di formulare ulteriori considerazioni riguardo le possibili vie di contaminazione. I risultati ottenuti hanno individuato 12 cluster formati da ceppi ad elevato livello di similitudine ( $\geq 90 \%$ ). Tre cluster $(5,6,7)$ sono costituiti da ceppi provenienti da impasti prelevati nello stesso sopralluogo, indicando la possibile contaminazione delle materie prime in entrata, come documentato da Peccio et al. (2003), poiché nessun sito ambientale è risultato contaminato da ceppi caratterizzati dallo stesso pulsotipo. Tre cluster $(4,9,10)$ hanno evidenziato una cross-contaminazione tra semilavorati e superfici in quanto raggruppano ceppi provenienti sia da impasti che dal pungibudello e il vagonetto. La stessa evidenza è stata osservata da Chen et al. (2010) in uno stabilimento di lavorazione di prodotti ittici. È importante sottolineare che certi ceppi, isolati a partire dall'ambiente, possono subire stress che non ne permettono la sopravvivenza una volta a contatto con gli alimenti oppure potrebbero non sopravvivere abbastanza a lungo sulle superfici per entrare in contatto con i prodotti (Destro et al., 1996). Tuttavia i ceppi isolati in questo studio hanno dimostrato di avere buone capacità di adattamento ai differenti substrati. I cluster 1,3,8 e 11 hanno evidenziato, oltre alla cross-contaminazione tra impasti, pungibudello e tritacarne, la presenza di ceppi persistenti: pulsotipi altamente correlati sono stati, infatti, isolati più volte durante il periodo considerato. Il cluster 2, inoltre, indica l'esistenza di ceppi persistenti unicamente di origine ambientale. I risultati ottenuti sono in linea con quanto riportato in differenti tipi di impianti da diversi autori, che hanno descritto periodi prolungati di persistenza. Miettinen $e t$ al. (1999), ad esempio, hanno osservato una persistenza di sette anni in un caseificio produttore di gelati, mentre Olsen et al. (2005) hanno isolato un ceppo di L. monocytogenes che ha persistito nello stesso impianto per almeno 12 anni e ha causato un focolaio epidemico a distanza di 11 anni dal primo episodio sporadico. Il cluster 3 è di particolare rilievo poiché mostra un'elevata similitudine $(92,4 \%)$ tra ceppi isolati da un impasto positivo per $L$. monocytogenes analizzato nell'ultima visita e quelli provenienti da impasti e pungibudelli campionati nei mesi precedenti. Questi rilievi potrebbero confermare l'ipotesi menzionata in precedenza di un reservoir localizzato nella prima parte dell'impianto, in grado di contaminare gli impasti nel corso di tutto il periodo di monitoraggio e che non è stato eliminato dalle procedure di sanificazione più accurate. La stessa ipotesi è stata formulata da studi svolti in precedenza: ad esempio, Ferreira et al.
(2011) hanno individuato nel primo locale di lavorazione l'origine della contaminazione, in seguito disseminata in tutta la catena di produzione. Tuttavia non è possibile escludere l'introduzione del microrganismo tramite le materie prime in ingresso, come discusso in precedenza. Infine, è stato identificato un ultimo cluster (12) composto da profili di ceppi provenienti dall'insaccatrice non correlati a pulsotipi di altra origine. Si tratta, probabilmente, di ceppi transitori introdotti nello stabilimento, ad esempio tramite le materie prime, ed eliminati dalle procedure di sanificazione, in quanto il loro isolamento è stato limitato ad un'unica visita (Chasseignaux et al., 2001).

La PFGE è una metodica di caratterizzazione dotata di un elevato potere discriminatorio che permette di rilevare l'elevata eterogeneità dei microrganismi isolati a partire da alimenti ed ambienti di lavorazione. In letteratura differenti autori hanno sottolineato come ceppi con pulsotipi diversi siano associati a persistenza nei salumifici; questo indica che molti ceppi sono in grado di stabilire contaminazioni persistenti negli impianti di produzione, determinando la successiva contaminazione dei salumi in essi prodotti (Ferreira et al., 2011; Felício et al., 2007). L'eterogeneità riscontrata può essere dovuta alla rapida evoluzione cui va incontro L. monocytogenes: è stato ipotizzato, infatti, che questa sia determinata dall'azione di profagi che permettono lo scambio di materiale genetico tramite trasduzione tra i diversi microrganismi. In seguito, i ceppi subiscono una naturale selezione volta a favorire quelli in grado di adattarsi al meglio all'ecologia dell'impianto produttivo in cui si trovano (Miettinen et al., 1999; Ferreira et al., 2011; Verghese et al., 2011).

\section{Conclusioni}

Questa ricerca evidenzia come sia indispensabile che i piani di sanificazione adottati dalle imprese debbano essere adattati alle peculiarità di ognuna di esse, indirizzando le attenzioni sulle criticità evidenziate dalle analisi svolte nell'ambito del piano di autocontrollo. Di conseguenza, occorrerebbe sensibilizzare i produttori nei confronti di un rigoroso monitoraggio di ambiente ed attrezzature, nonché nell'applicazione delle metodiche di tipizzazione che di norma non sono neppure contemplate dato il costo e la limitata disponibilità di laboratori in grado di eseguirle. La conoscenza dettagliata dei ceppi che circolano nel/i locale/i di produzione o che sono introdotti dalle materie prime sarebbe utile al fine di limitare le spese in termini di applicazione delle misure correttive, come la mancata commercializzazione di prodotti non conformi ai criteri di legge oppure operazioni di ritiro e richiamo.
Oltre all'utilità nell'ambito del monitoraggio, l'applicazione su larga scala delle metodiche di caratterizzazione potrebbe permettere l'istituzione di un database nazionale che raccolga i profili rilevati, associati alle informazioni riguardanti il periodo e la fonte di isolamento. Tutto ciò permetterebbe di identificare le fonti di trasmissione e, in caso di epidemie, di indirizzare le indagini epidemiologiche tradizionali, aumentando così le probabilità di arrivare tempestivamente all'identificazione dell'alimento responsabile dell'epidemia.

\section{Bibliografia}

Autio T, Hielm S, Miettinen M, Sjöberg AM, Aarnisalo K, Björkroth J, MattilaSandholm T, Korkeala H, 1999. Sources of Listeria monocytogenes contamination in a cold-smoked rainbow trout processing plant detected by Pulsed-Field Gel Electrophoresis typing. Appl Environ Microb 1:150-5.

Chasseignaux E, Toquin M-T, Ragimbeau C, Salvat G, Colin P, Ermel G, 2001. Molecular epidemiology of Listeria monocytogenes isolates collected from the environment, raw meat and raw products in two poultryand pork-processing plants. J Appl Microbiol 91:888-99.

Chen B-Y, Pyla R, Kim T-J, Silva JL, Jung YS, 2010. Prevalence and contamination patterns of Listeria monocytogenes in cat fish processing environment and fresh fillets. Food Microbiol 27:645-52.

Commissione Europea, 2002. Regolamento del Parlamento Europeo e del Consiglio del 28 gennaio 2002 che stabilisce i principi e i requisiti generali della legislazione alimentare, istituisce l'Autorità europea per la sicurezza alimentare e fissa procedure nel campo della sicurezza alimentare, 178/2002/CE. In: Gazzetta Ufficiale, L 31, $1 / 2 / 2002$.

D’Agostino M, Wagner M, Vazquez-Boland JA, Kuchta T, Karpiskova R, Hoorfar J, Novella S, Scortti M, Ellison J, Murray A, Fernandes I, Kuhn M, Pazlarova J, Heuvelink A, Cook N, 2004. A validated PCR method to detect Listeria monocytogenes using raw milk as a food modeltowards an international standard. J Food Protect 67:1646-55.

Destro MT, Leitão MFF, Farber JM, 1996. Use of molecular typing methods to trace the dissemination of Listeria monocytogenes in a shrimp processing plant. Appl Environ Microb 2:705-11.

Donlan RM, 2001. Biofilm formation: a clinically relevant microbiological process. Clin Infect Dis 33:1387-92.

Felício MTS, Hogg T, Gibbs P, Teixeira P, 
Wiedmann M, 2007. Recurrent and sporadic Listeria monocytogenes contamination in Alheiras represents considerable diversity, including virulence-attenuated isolates. Appl Environ Microb 73:3887-95.

Ferreira V, Barbosa J, Stasiewicz M, Vongkamjan K, Moreno Switt A, Hogg T, Gibbs P, Teixeira P, Wiedmann M, 2011. Persistent Listeria monocytogenes in fermented meat sausage production facilities in Portugal represent diverse geno- and phenotypes. Appl Environ Microb 77:270115.

Gandhi M, Chikindas ML, 2007. Listeria: a foodborne pathogen that knows to survive. Int J Food Microbiol 113:1-15.

Giovannacci I, Ragimbeau C, Queguiner S, Salvat G, Vendeuvre JL, Carlier V, Ermel G, 1999. Listeria monocytogenes in pork slaughtering and cutting plants use of RAPD, PFGE and PCR-REA for tracing and molecular epidemiology. Int $\mathrm{J}$ Food Microbiol 53:127-40.

Graves LM, Swaminathan B, 2001. PulseNet standardized protocol for subtyping Listeria monocytogenes by macrorestriction and pulsed-field gel electrophoresis. Int J Food Microbiol 65:55-62.

ISO, 2005. Microbiology of food and animal feeding stuffs. Horizontal method for the detection and enumeration of Listeria monocytogenes. Part 1: detection method. Norma UNI EN ISO 11290-1. Organizzazione internazionale per la normazione ed., Ginevra, Svizzera.

Kabuki DY, Kuaye AY, Wiedmann M, Boor KJ, 2004. Molecular subtyping and tracking of Listeria monocytogenes in latin-style fresh-cheese processing plants. J Dairy Sci 87:2803-12.

Leroy F, Verluyten J, De Vuyst L, 2006. Functional meat starter cultures for improved sausage fermentation. Int J Food Microbiol 106:270-85.

Lomonaco S, Decastelli L, Nucera D, Gallina S, Manila Bianchi D, Civera T, 2009. Listeria monocytogenes in Gorgonzola: subtypes, diversity and persistence over time. Int $\mathrm{J}$ Food Microbiol 128:516-20.

Low JC, Donachie W, 1997. A review of Listeria monocytogenes and listeriosis. Vet $\mathrm{J}$ 153:9-29.

Miettinen MK, Björkroth KJ, Korkeala HJ, 1999. Characterization of Listeria monocytogenes from an ice cream plant by serotyping and pulsed-field gel electrophoresis. Int J Food Microbiol 46:18792.

Olsen SJ, Patrick M, Hunter SB, Reddy V, Kornstein L, MacKenzie WR, Lane K, Bidol S, Stoltman GA, Frye DM, Lee I, Hurd S, Jones TF, LaPorte TN, DeWitt W, Graves L, Wiedmann M, Schoonmaker-Bopp DJ, Huang AJ, Vincent C, Bugenhagen A, Corby J, Carloni ER, Holcomb ME, Woron RF, Zansky SM, Dowdle G, Smith F, AhrabiFard S, Ong AR, Tucker N, Hynes NA, Mead P, 2005. Multistate outbreak of Listeria monocytogenes infection linked to delicatessen turkey meat. Clin Infect Dis 40:962-7.

Palmer J, Flint S, Brooks J, 2007. Bacterial cell attachment, the beginning of a biofilm. J Ind Microbiol Biot 34:577-88.

Pan Y, Breidt F Jr, Kathariou S, 2006. Resistance of Listeria monocytogenes biofilms to sanitizing agents in a simulated food processing environment. Appl Environ Microb 12:7711-7.

Peccio A, Autio T, Korkeala H, Rosmini R, Trevisani M, 2003. Listeria monocytogenes occurrence and characterization in meatproducing plants. Lett Appl Microbiol 37:234-8.
Reij M, De Aantrekker ED, 2004. Recontamination as a source of pathogens in processed foods. Int $\mathrm{J}$ Food Microbiol 91:1-11.

Robbins JB, Fisher CW, Moltz AG, Martin SE, 2005. Elimination of Listeria monocytogenes biofilms by ozone, chlorine, and hydrogen peroxide. J Food Protect 68:4948.

Samelis J, Metaxopoulos J, Vlassi M, Pappa A, 1998. Stability and safety of traditional Greek salami: a microbiological ecology study. Int J Food Microbiol 44:69-82.

Thévenot D, Delignette-Muller ML, Christieans S, Vernozy-Rozand C, 2005. Prevalence of Listeria monocytogenes in 13 dried sausage processing plants and their products. Int J Food Microbiol 102:85-94.

Tompkin RB, 2002. Control of Listeria monocytogenes in the food-processing environment. J Food Protect 4:709-25.

Verghese B, Lok M, Wen J, Alessandria V, Chen Y, Kathariou S, Knabel S, 2011. comK prophage junction fragments as markers for Listeria monocytogenes genotype unique to individual meat and poultry processing plants and a model for rapid nichespecific adaptation, biofilm formation and persistence. Appl Environ Microb 77:327992.

Weatherill S, 2009. Report of the independent investigator into the 2008 listeriosis outbreak. Health Canada 2009. Disponibile al sito: http://www.listeriosis-listeriose. investigation-enquete.gc.ca/lirs_rpt_e.pdf

WHO/FAO, 2004. Risk assessment of Listeria monocytogenes in ready-to-eat foods. WHO ed., Ginevra, Svizzera. Disponibile al sito: http://www.who.int/foodsafety/publications/micro/mra_listeria/en/ 